QUADERNS DE FILOSOFIA VOL. IV NÚM. I (2017): IO7-I 8

eISSN: 234I-3042 DOI: I 0.7203/QFIA.4.I.946I

Carla Carreras

Universitat de Girona

\title{
Del homo ludens a la gamificación
}

(From Homo Ludens to gamification)

Recibido: 31/12/16. Aceptado: 25/3/17

Resumen: Parece que las últimas tendencias en educación pasan por introducir la gamificación en las aulas y en todas las actividades educativas. La idea de que los contenidos deben interesar a nuestros estudiantes combinada con el convencimiento de que "se aprende mejor jugando", han llevado a pensar que debemos dar a esos contenidos una forma lúdica o, mejor todavía, convertirlos en juegos. ¿Es la gamificación una moda o una oportunidad? Y en el caso concreto de la educación, ¿se trata sólo de un recurso metodológico para aprender más eficazmente o es una característica que debería tener toda actividad que pretenda ser educativa? ¿Cómo gamificar las clases (de filosofía, en nuestro caso)?

\begin{abstract}
Latest trends in education go through introducing gamification in classrooms and in all educational activities. The idea that contents should interest our students combined with the conviction that "they learn better by playing" have led us to think that we should give these contents a playful form or, even better, turn them into games. Is gamification a trend or an opportunity? And in the specific case of education, is it only a methodological resource to learn more effectively or is it a characteristic that should have all activity that pretends to be educational? How to gamify courses (philosophy courses, more concretely)?
\end{abstract}

Palabras clave: gamificación, Huizinga, Homo ludens.

Keywords: gamification, Huizinga, Homo ludens. 
$\mathrm{E}$ N I938, el historiador holandés Johan Huizinga publicó un libro, Homo ludens, en el que defendía que las designaciones de homo sapiens y homo faber aplicadas a nuestra especie eran claramente insuficientes. Huizinga (I972, 7) afirmaba que "el nombre de homo ludens, el hombre que juega, expresa una función tan esencial como la de fabricar, y merece, por tanto, ocupar su lugar junto al de homo faber" en la caracterización de la especie humana. Con esta declaración de intenciones, Huizinga reivindicaba el juego como fenómeno cultural (y no como una simple función biológica). Casi un siglo después, en la era de los videojuegos, hemos empezado a hablar de gamificación (o ludificación, en las lenguas románicas) ${ }^{1}$ para referirnos no solo al "uso de los elementos y de la mecánica del juego en contextos ajenos al mismo" sino también a "hacer vivir experiencias de aprendizaje gratificantes usando elementos de juego". ¿De qué manera la gamificación confirma (si es que lo hace) los análisis de Huizinga? ¿Es la gamificación una moda o una oportunidad? Y en el caso concreto de la educación, ¿se trata solo de un recurso metodológico para aprender más eficazmente o es una característica que debería tener toda actividad que pretenda ser educativa? ¿Cómo gamificar las clases (de filosofía, en nuestro caso)?

\section{I. ¿QUÉ ES LA GAMIFICACIÓN?}

Según Imma Marín Santiago, ${ }^{2}$ Mary Poppins fue la primera precursora de la gamificación; en la canción $A$ Spoonful of sugar canta:

In every job that must be done

There is an element of fun

You find the fun and ... snap! the job 's a game

${ }^{1}$ La palabra inglesa gamification deriva de la palabra game, que significa 'juego'. De manera análoga, la acepción en español, ludificación, proviene de la palabra latina ludus, ludere ('juego', 'jugar'). El DRAE no recoge todavía ninguno de los dos términos. En catalán, el Termcat (centro de terminología de la lengua catalana, creado en 1985 por la Generalitat de Catalunya y el Institut d'Estudis Catalans) ha optado por el término 'ludificació'. Sin embargo, el término más habitual en la red es el de 'gamificación', que es el que utilizaré en este artículo.

Ya en 1938, Huizinga observa que "ludus, ludere como concepto general de juego y jugar, no solo no ha pasado a los idiomas románicos, sino que, por lo que veo, apenas ha dejado ninguna huella. En todas las lenguas romances, los vocablos iocus, iocari han ampliado su ámbito significativo al terreno del juego y del jugar relegando completamente ludus, ludere" (Huizinga I 972 , 55). En español, aunque 'juego' deriva de iocus, hay palabras relacionadas con el juego que proceden de ludus, p.e. 'lúdico' o la misma 'ludificación'.

2 Imma Marín Santiago, Gamificación en educación. [https://www.youtube.com/ watch?v=qEYP9hrSRXc, Consulta: 19 de mayo de 2016] 
And every task you undertake becomes a piece of cake

A Lark! a spree!

It 's very clear to see

... That a spoonful of sugar...

En esta escena de la película, de 1964, el objetivo de Mary Poppins es hacer ver a los hermanos Banks que ordenar la habitación también puede ser una tarea divertida. De hecho, toda la película es una reivindicación del lado lúdico de la vida en contra de la seriedad con que el padre de familia se toma todas las cosas: el trabajo, la familia.

¿En esto consiste la gamificación? ¿Es cierta la afirmación de Mary Poppins según la cual "cualquier trabajo (o aprendizaje) tiene algo de divertido"? ¿Podemos gamificarlo todo?

La primera vez que se utiliza la palabra gamification es en 2003, cuando Nick Pelling funda la consultora Conundra, que ofrece precisamente utilizar las mecánicas de juego como recurso para vender productos de consumo. Así, la gamificación aparece por primera vez aplicada al mundo del marketing, pero se ha extendido con rapidez a otros sectores, como la educación, la cultura o la investigación científica.

Según la definición clásica, la gamificación es el "uso de los elementos y de la mecánica del juego en contextos ajenos al mismo, con el objetivo de orientar el comportamiento de las personas y conseguir determinadas metas", como estimular el interés, incentivar un cambio de comportamiento o transmitir un mensaje o contenido. Oriol Ripoll propone una alternativa a esta definición que incide no tanto en los recursos utilizados (los elementos y las mecánicas del juego) como en el resultado: "Hacer vivir experiencias de aprendizaje gratificantes usando elementos de juego". La definición de Ripoll tiene la ventaja de sustraer la gamificación del ámbito puramente del marketing y situarla en el campo de las experiencias de aprendizaje con el objetivo de conseguir que estas sean gratificantes. No se trata tanto de "orientar el comportamiento de las personas" como de estimular el interés por aprender.

La gamificación se entiende a menudo como el uso de los elementos y de la mecánica de los videojuegos. Sin embargo, Ripoll insiste en la idea de que la gamificación no debe circunscribirse exclusivamente al ámbito de los videojuegos (on-line y/o off-line), sino que debe entenderse de manera amplia y abarcar también necesariamente todo el ámbito de los juegos de mesa (tradicionales y modernos) y también los juegos de calle, los juegos de rol, juegos de competición, etc. En este sentido, pone de relieve la capacidad del juego — en general, y no sólo de los tecnológicos - para estimular la atención y la implicación de los individuos en las tareas que realizan. 
La gamificación no debe confundirse con la "teoría de los juegos", una teoría que "analiza matemáticamente el comportamiento óptimo de varios jugadores ante las posibles estrategias aplicables para la resolución del juego". ${ }^{3}$ Un ejemplo muy conocido de la aplicación de la teoría de juegos en la vida real es el "dilema del prisionero", popularizado por el matemático Albert W. Tucker, que tiene muchas implicaciones para comprender la naturaleza de la cooperación humana. La teoría de los juegos es, pues, un análisis de conductas y elecciones y no tiene que ver con utilizar elementos del juego para convertir un trabajo en una "experiencia gratificante".

En cualquier caso, según las definiciones apuntadas, parece que en la mayoría de los casos el juego se entiende como un recurso para conseguir un resultado. Esta visión es diferente de la que propone Imma Marín. Ella lamenta esta visión del juego porque es parcial y por ello remite a Mary Poppins: "Cualquier tarea tiene una parte divertida"; esto significa que el juego no es solo una técnica, una metodología aplicada o aplicable a una tarea determinada, sino que es un aspecto que forma parte de esa tarea. Marín habla también de "actitud vital" ante la tarea. En este sentido, el aspecto lúdico no sería solo un aspecto de la tarea, sino también la manera en que nosotros la encaramos (precisamente esta actitud nuestra sería la que hace posible captar el elemento lúdico en todo aquello que no llamamos juego). Desde este punto de vista, pues, la gamificación no es solo "aplicar" las mecánicas del juego en contextos ajenos a él, sino que sería, más bien, la identificación de aquel elemento lúdico que puede convertir una tarea en un juego. El planteamiento de Marín exige, sin embargo, un análisis más detallado de la exactitud de la afirmación de Mary Poppins y de las posibilidades (o no) de gamificarlo todo.

Huizinga (1972, 45) define el juego como "una acción libre ejecutada 'como si' y sentida como situada fuera de la vida corriente, pero que, sin embargo, puede absorber completamente al jugador, sin que haya en ella ningún interés material ni se obtenga ningún provecho, que se ejecuta dentro de un determinado tiempo y un determinado espacio, que se desarrolla en un orden sometido a reglas y que da origen a asociaciones que tienden a rodearse de misterio para disfrazarse y para destacarse del mundo habitual."

Así, identifica una serie de características esenciales del juego: ser voluntario; tener límites espaciales y temporales (un paréntesis en la vida "corriente"); ser desinteresado; y tener un sistema de reglas. Jane McGonigal señaló que los puntos clave de la gamificación son un objetivo claro, una retroalimentación rápida y la participación voluntaria. Aunque McGonigal remite a Huizinga, aquí ya vemos alguno de los puntos que distinguen el análisis del

${ }^{3}$ DIEC, “Teoria dels jocs" [consulta: 16 mayo 2016]. 
juego de Huizinga y la propuesta gamificadora: de entrada, el objetivo claro y la retroalimentación.

\section{LA GAMIFICACIÓN EN LA EDUCACIÓN: OPORTUNIDADES Y RIESGOS}

Se podría decir que, desde siempre, el ámbito de la educación se ha servido de los recursos lúdicos: las cantinelas que acompañaban a las tablas de multiplicar o las listas que había que aprender en la escuela, tales como las de reyes, capitales o accidentes geográficos, eran también recursos supuestamente lúdicos para facilitar la memorización. En todos los casos, sin embargo, incumplían los requisitos de la definición de Ripoll en la medida en que su objetivo no era "lograr experiencias gratificantes de aprendizaje" sino la memorización o consecución de un determinado conocimiento. Desde hace unos años, no es extraño encontrar maestros y profesores que utilizan pequeños concursos o juegos para estimular y facilitar el aprendizaje a sus alumnos; en este sentido, se habla del juego como recurso educativo. En la época de los juegos "tecnológicos" y viendo la atracción que los videojuegos y las apps despiertan en los niños y los jóvenes, desde la educación se ha planteado con insistencia la necesidad de adaptar la enseńanza utilizándolas como recurso educativo.

En el contexto actual los jóvenes pasan gran parte de su tiempo jugando a videojuegos o con las tecnologías de la información y la comunicación. Muchos estudios han destacado las habilidades que desarrollan estas tecnologías, por lo que no debería verse como algo extraño la introducción de las mecánicas y las dinámicas de juego de los videojuegos en el proceso de aprendizaje para aprovechar precisamente estas habilidades, incentivando al mismo tiempo el interés y la participación activa de los estudiantes. En esta línea va la defensa de la introducción del móvil o el portátil en las aulas, no como sustitutos del libro de texto (utilizando los libros digitales o las enciclopedias en red) sino utilizando los juegos y las apps como recurso pedagógico. Hay muchos juegos - no solo videojuegos - que pueden aplicarse en un aula, desde el concurso más básico hasta juegos multijugador a través de los móviles inteligentes.

Sin embargo, para Imma Marín, es simplificador hablar del juego como un recurso más entre todos los demás; según ella, no se trataría tanto de aplicar el juego como metodología como de buscar aquellos elementos dentro de las tareas que hay que hacer que puedan ser gratificantes. A menudo se apela a la búsqueda de sentido, a la experimentación individual (los experimentos en el laboratorio) o la aplicación práctica (p.e. ver para qué puede servir aprender las tablas) como elementos que incrementan el interés de los alumnos para las actividades escolares; pero esto no es suficiente. Algunas de estas cosas pueden, 
además, tener una forma más lúdica, más atractiva: los experimentos en el laboratorio, por ejemplo, pueden ser planteados como un juego.

Una concreción de la gamificación son los llamados “juegos serios” (serious games), videojuegos desarrollados no con el propósito de que resulten entretenidos, sino con una finalidad educativa o formativa. Así, no toda gamificación consiste en un "juego serio" pero sí podríamos considerar que todo "juego serio" es una actividad de gamificación en la medida en que comparte los objetivos de esta, entendida según la definición de Ripoll: el "juego serio" puede entenderse como un desarrollo tecnológico de aquella preocupación pedagógica que comparten muchos docentes por la necesidad de incorporar a sus clases un componente motivador y estimulante que garantice la atención y el interés de sus estudiantes. Según esto, la gamificación aplicada a la educación tomaría la forma de un serious game, en la medida en que tiene una finalidad concreta: estimular la atención e implicación de los estudiantes y convertir el aprendizaje en una experiencia altamente significativa. Parece que con el añadido del calificativo "serio", existe la intención de "dignificar" la utilización del juego en las aulas, como si hablar simplemente de "juego" implicara banalidad y superficialidad.

En Homo ludens, Huizinga comenta precisamente la contraposición que suele hacerse entre el juego y lo serio. Según él, "juego" y "lo serio" "no son términos equivalentes: "Lo serio trata de excluir el juego, mientras que el juego puede muy bien incluir en sí mismo lo serio" (Huizinga i 972, 27). Para Huizinga, podemos tomarnos (y a menudo lo hacemos) seriamente el juego en el que participamos, y esto se muestra tanto en el seguimiento de las reglas como en el embeleso (o la intensa concentración) que a veces (nos) provoca el juego.

Hemos dicho que una de las características de la gamificación es la participación voluntaria (y en esto coinciden Huizinga y McGonigal). En la gamificación de las actividades escolares y académicas, esta participación voluntaria es un factor que se pone en cuestión en la medida en que utilizamos el juego (o algunos de sus elementos) para realizar actividades de participación o de evaluación no optativas. Aunque la libre participación se ha presentado como una característica necesaria del juego, en el planteamiento gamificador de las aulas ha pasado a ser una característica secundaria. Habría que reflexionar si esto llega a poner en cuestión el carácter lúdico de la actividad gamificada: ¿¿deja de ser un juego? Parece que, si seguimos la definición de Huizinga, perder la condición de voluntario es perder también la condición de "juego". Sin embargo, tal vez podría argumentarse que, a pesar de no ser estrictamente un juego, puede

\footnotetext{
${ }^{4}$ Huizinga también comenta que "la oposición complementaria 'el juego/lo serio' no siempre se expresa con dos palabras de manera tan perfecta como en los idiomas germánicos" (HuIZINGA I $972,64-5)$. En castellano, p.e., no hay una palabra para referirse a "lo serio".
} 
mantener un cierto carácter lúdico. Y, de hecho, la gamificación no se presenta como el uso de juegos sino como "el uso de los elementos y de la mecánica del juego en contextos ajenos al mismo"; en este sentido, pues, el objetivo no es tanto ofrecer juegos como actividades educativas como aprovechar algunos de los elementos más gratificantes de aquellas actividades que llamamos juegos en el caso de tareas y trabajos que no son —ni queremos que sean- juegos.

En la gamificación se distingue entre mecánicas y dinámicas del juego: "Las mecánicas del juego son las diferentes acciones, comportamientos, técnicas y mecanismos de control que se utilizan para convertir en juego una actividad. Se trata de los aspectos que, en conjunto, crean una experiencia atractiva y de fácil adhesión para el jugador. Por otra parte, la dinámica de juego es el efecto, motivación y/o deseos que se consiguen o se desea conseguir en el usuario".5

Algunas mecánicas propias de los juegos son: la consecución de éxitos o los sistemas de puntuación, los avatares, los niveles, los desafíos, etc. Las dinámicas de juego son, por su parte, igualmente variadas y van desde fomentar una sana competición entre los alumnos hasta la necesidad de expresar la autonomía y la propia identidad. En la definición clásica, se habla del uso de los elementos y de la mecánica del juego; Oriol Ripoll, en cambio, pone más énfasis en la dinámica: lograr experiencias gratificantes.

Hay diferentes maneras de aplicar la gamificación a una asignatura atendiendo a las diferentes mecánicas y a las diversas dinámicas. También hay que tener en cuenta las actividades que hay que realizar y los objetivos que se pretende conseguir. Algunas de las estrategias más importantes son:

a) La elaboración de una historia, narrativa o universo (más o menos sofisticado) diseñado de manera atractiva que funcione de hilo temático de todas las actividades (a modo de centro de interés). Lipman (I994, 145) afirmaba que "los niños se ven más inclinados a aprender cuando sus textos [o actividades] tienen forma narrativa".

b) La introducción de elementos de personalización: desde la especialización de cada miembro del grupo en los trabajos colectivos hasta la elección de avatares o personajes de la historia.

c) Los sistemas de retroalimentación: el feedback inmediato y constante facilita que el alumno sepa en todo momento cuál es su estado de aprendizaje y pueda autocorregirse mejor y más rápidamente.

d) Las misiones y retos: estimulan el interés y ponen en juego todas las habilidades de pensamiento dirigidas a superar las "pantallas" y los niveles.

e) Los desafíos: es una estrategia muy habitual que estimula el interés en base a la competitividad entre los participantes.

\footnotetext{
${ }^{5}$ http://www.gamificacion.com/claves-de-la-gamificacion [Consulta: 20 mayo 2016].
} 
f) La interactividad: los juegos ponen "en juego" (valga la redundancia) una serie de habilidades en red, sin compartimentarlas en disciplinas estancas, lo que facilita que sean percibidos como una experiencia significativa en su conjunto.

g) Los sistemas de premio. Pueden tener diferentes formas: puntos extra (los alumnos son estimulados a trabajar a cambio de una pequeña subida de la calificación obtenida de forma instantánea con puntos extra o un pequeño porcentaje de la nota); bienes virtuales (indumentaria propia, armas, monedas, accesorios...); acceso a niveles superiores, etc.

Está claro que los juegos o actividades lúdicas no utilizan todas las mecánicas a la vez: hay juegos de estrategia, juegos narrativos, juegos de habilidad, etc. No parece, sin embargo, que haya duda de que estas mecánicas obtienen unos beneficios evidentes en el aprendizaje: la retroalimentación es inmediata, y la aceptación del error como elemento connatural al conocimiento y al crecimiento elimina gran parte del miedo del estudiante al fracaso. La interactividad es un elemento extra de motivación, en la medida en que engloba todos los aspectos en una unidad de significación (es la base también del trabajo por proyectos, por ejemplo): "Los significados surgen cuando percibimos la relación entre la parte y el todo" (Lipman i99i, 29).

Sin embargo, para que el análisis sea completo y podamos contemplar el potencial de la gamificación de una manera más efectiva, hay que revisarla a la luz de los posibles riesgos, a la manera de un análisis DAFO. ${ }^{6}$ En este sentido, no es suficiente con señalar las bondades de un planteamiento lúdico en educación (bondades que pueden ser más o menos evidentes para todos) sino que hay que plantear cuáles son las amenazas o las debilidades que una concepción lúdica en educación puede conllevar. No se trata en ningún caso de despreciar este componente lúdico sino de ser conscientes de los límites y de todo aquello que puede hacer caer la propuesta educativa del lado de la banalización o de la excesiva simplificación. Desde esta perspectiva, es necesario no perder de vista el objetivo de la gamificación: crear experiencias de aprendizaje gratificantes. Se trata de aprender integrando este aprendizaje en nuestra experiencia, para hacerla mejor y más significativa; es decir, la incorporación del juego (de sus mecánicas y de sus dinámicas) debe ser una oportunidad para convertir un trabajo tedioso en un reto interesante.

Las debilidades de la propuesta gamificadora tienen que ver, en nuestra opinión, con el objetivo, tal como lo expresa la definición clásica: "Orientar el comportamiento de las personas y conseguir determinadas metas". Orientar la

${ }^{6}$ El análisis DAFO, también conocido como análisis FODA o DOFA, es una metodología de estudio de la situación de una empresa o un proyecto, analizando sus características internas (Debilidades y Fortalezas) y su situación externa (Amenazas y Oportunidades ) en una matriz cuadrada. Proviene de las siglas en inglés SWOT (Strengths, Weaknesses, Opportunities y Threats). 
conducta es solo un objetivo formal que todavía hay que dotar de contenido. Cuando McGonigal habla de la necesidad de "tener un objetivo claro" se refiere más bien a la finalidad hacia la que quisiéramos "orientar la conducta", es decir, la dirección que debe tomar en función de la finalidad. Y en este punto, el hecho de que el término gamification tenga su origen en el ámbito del marketing ya da idea de algunos de estos "objetivos": comprar determinados productos de consumo, justificar ciertas opiniones, etc. La definición de Ripoll ayuda un poco a matizar el objetivo de la gamificación: "Hacer vivir experiencias gratificantes", pero no evita el riesgo. Las amenazas externas vienen también de un cierto uso "mercenario" que se pueda hacer, aprovechando precisamente esta debilidad.

\section{LA GAMIFICACIÓN EN LAS CLASES DE FILOSOFÍA: ALGUNOS EJEMPLOS}

Las clases de filosofía son, en algunos aspectos, similares a las clases de las otras asignaturas. En otros aspectos, sin embargo, son muy diferentes. Aquí aparece la cuestión de la didáctica: ¿Hasta qué punto la didáctica de la filosofía es igual, similar o diferente de las otras didácticas especiales? No desarrollaremos este punto en este momento pero sí queremos subrayar que las respuestas que damos a esta pregunta inciden directamente en la cuestión de la gamificación en las clases de filosofía.

En nuestra opinión, los tempos en las clases de filosofía deberían ser lentos (decimos deberían porque no suelen serlo, perseguidos como estamos por los contenidos de los programas y sus evaluaciones). Esto influye enormemente en los tipos de mecánicas de juego que podrían ser adecuadas. Esto no impide que, en algún momento, podamos hacer uso de mecánicas más rápidas o competitivas, pero siempre hay que tener muy clara la finalidad para la que utilizamos uno u otro procedimiento.

El artículo de David Medina (2015) "Poner cera, pulir cera' (filosofía y gamificación)" muestra algunas de las actividades que pueden realizarse en la clase de filosofía. Medina expone dos experiencias que realizó con sus estudiantes con Kahoot! (una aplicación de preguntas y respuestas tipo test) y Quandary (un serious game cuyo objetivo es resolver diversas situaciones conflictivas). Está claro que los dos ejemplos responden a actividades con objetivos muy diferentes y no se pueden generalizar a todas las actividades de la clase de filosofía. Kahoot!, por ejemplo, presenta las mismas limitaciones que plantea el clásico examen tipo test, de utilización muy restringida en la asignatura de filosofía. Es cierto que el entorno es más atractivo y se ofrece a través del móvil (un aparato a menudo proscrito en las aulas), pero lo que hay que analizar es si el envoltorio tecnológico justifica (y hasta qué punto y en qué casos) o no justifica la utiliza- 
ción de esta aplicación. En cuanto a Quandary, el hecho de ser un serious game ya parece que le confiere una característica especial, en la medida en que ha sido diseñado con fines abiertamente educativos, y por tanto parece también que se justifica su uso. Lo que habría que plantearse es si la utilización de estas "herramientas" garantiza el interés y el deseo de significación y si realmente nos acerca al objetivo de la asignatura. Seguramente sí logramos el objetivo de tener una "experiencia más gratificante" para los alumnos, pero también hay que valorar si se corresponde con una mejora de los conocimientos o con una experiencia más significativa.

En otro artículo, José Carlos Cortizo y sus colegas (20 I I) presentan una propuesta metodológica para la enseñanza universitaria que puede adaptarse a la asignatura de filosofía; el objetivo principal es conseguir "motivar a los alumnos, favorecer la competitividad, así como ofrecer un feedback instantáneo al estudiante, de modo que en todo momento pueda equipararse con el resto de sus compañeros, y también tener una idea clara de su posible nota final". Una de las mecánicas que proponen es la que han titulado "Tranquilo, esto lo soluciono yo", asociada a la colaboración entre personas o equipos, por la que se da puntos no solo a quien realiza el trabajo sino también a quien le ayuda (esta mecánica recuerda el sistema de puntuación del Dixit, un juego de cartas).

En todos estos ejemplos se ve como no se trata tanto de jugar como de elegir aquellos elementos de los juegos que pueden resultar interesantes para hacer de la clase una "experiencia más gratificante".

Matthew Lipman comenta que "todo lo que nos ayude a descubrir el sentido de la vida es educativo y las escuelas son educativas sólo en el caso de que faciliten este descubrimiento" (Lipman; Sharp y Oscanyan i992, 27). Análogamente, ¿podemos decir que una experiencia educativa debe ser gratificante, además de significativa? Si la respuesta es sí, entonces "el uso de elementos y mecánicas de juego" en contextos educativos deja de ser un recurso para pasar a ser una característica que debería tener toda educación, y la gamificación debería verse como una oportunidad y no como una moda.

\section{Huizinga y la gamificación}

Queda por ver si la gamificación puede ser vista como un paso adelante en la idea de Huizinga de caracterizar al ser humano como homo ludens y el juego como fenómeno cultural.

Es evidente que el juego (sea videojuego o juego tradicional, on-line u off-line) forma parte de la cultura del siglo xxi. Según Huizinga (I972, 45), "el juego es una actividad u ocupación libre, que se desarrolla dentro de unos 
límites temporales y espaciales determinados, según reglas absolutamente obligatorias, aunque libremente aceptadas, acción que tiene su fin en sí misma y que va acompañada de un sentimiento de tensión y alegría y de la conciencia de 'ser de otro modo' que en la vida corriente."

En este sentido, los juegos del siglo XXI (tecnológicos o no) siguen las características de la definición de Huizinga. Seguramente, los juegos de rol y la realidad virtual han elevado en un grado inimaginable por Huizinga la última característica de "ser de otra manera".

Huizinga (1972, 34) relaciona el juego con el culto, con lo sagrado: "Los conceptos de ritos, magia, liturgia, sacramento y misterio entrarían en el campo del concepto 'juego'." Los juegos modernos participan de este universo de significado. Huizinga ( 1972,34$)$ no se refiere en ningún caso a la banalización de lo sagrado, sino al revés: el juego es también fenómeno cultual: "En esta identificación platónica del juego y lo sagrado, lo sagrado no desmerece para que se lo califique de juego, sino que éste queda exaltado porque su concepto se eleva hasta las regiones más altas del espíritu." Es lo que queremos decir, de hecho, cuando hablamos de "series de culto" o de la "liturgia" o los "rituales" de los juegos de rol. A primera vista, puede parecer, ciertamente, una banalización de los conceptos, pero Huizinga defendería que, en el fondo, el embeleso ante el misterio, en uno y otro caso, es similar.

¿Cómo se relaciona todo esto con la gamificación? En nuestra opinión, no hay mucha relación. La gamificación parte de un planteamiento finalista que la aleja de este embeleso. Lo que busca es otra cosa: conseguir determinadas metas o hacer vivir experiencias gratificantes de aprendizaje. No es que sean objetivos banales ni indeseables, ciertamente, pero las actividades gamificadas no cumplen los requisitos de ser una ocupación libre, desinteresada, ni de tener aquella conciencia de "ser de otra manera". Ya hemos visto, también, como McGonigal señalaba que, a diferencia de la definición de Huizinga, tener un objetivo claro y una retroalimentación rápida eran los puntos clave de la gamificación. Huizinga y McGonigal solo coinciden en que debe ser una participación voluntaria, pero este requisito también queda en suspenso en muchos de los casos concretos de gamificación.

De esta manera, tal vez podamos concluir que aunque el juego, tal como lo define Huizinga, está presente (quizás más que nunca) en la cultura del siglo XXI, la gamificación no es un argumento más a favor de esta afirmación sino una oportunidad educativa. Las mismas definiciones de gamificación ya reconocen que el objetivo es aplicar o utilizar las mecánicas del juego, y no convertir las aulas (o el espacio del que se trate) en una "comunidad de juego". 


\section{BIBLIOGRAFÍA}

Carrillo, N. 2013, "Cap a la ludificació: viure jugant", Diari ARA, 2/12/2013 [http://www.ara.cat/premium/Cap-ludificacio-viure-jugant_0_1040296049. html, Consulta: 23 mayo 2016].

Cortizo Pérez, J. C. et al. 20 i I, "Gamificación y Docencia: Lo que la Universidad tiene que aprender de los Videojuegos”, VIII Jornadas Internacionales de Innovación Universitaria, Villaviciosa de Odón, 11-12 julio 2011 [http://www. josek.net/publicaciones/JIU2011-Preprint.pdf, Consulta: 23 mayo 2016].

Gallego, F. J. et al. 20 I4, "Panorámica: serious games, gamification y mucho más", ReVisión, vol. 7, núm. 2, 2014 [http://www.aenui.net/ojs/index.php?jo urnal=revision $\&$ page=article $\& o p=$ view $\&$ path []$=148 \&$ path []$=249$, Consulta: 20 mayo 2016].

Huizinga, J. I972, Homo ludens, Madrid: Alianza Editorial.

Lipman, M. I994, "Fortalecer el razonamiento y el juicio por medio de la filosofía”, en Maclure, S. y Davies, P., Aprender a pensar, pensar en aprender, Barcelona: Gedisa, 143-54.

Lipman, M.; Sharp, A. M. y Oscanyan, F. i992, Filosofia a l'escola, Vic: Eumo, (Traducción de Eulàlia Presas). [Philosophy in the Classroom, Philadelphia: Temple University Press, I977.]

Marczewski, A. 20 I2, Gamification: A Simple Introduction, [Consulta: 15 mayo 2016].

Marín Santiago, I. Gamificación en educación, [https://www.youtube.com/ watch?v=qEYP9hrSRXc, Consulta: 19 mayo 2016].

McGonigal, J. 20 I I, Reality is Broken. Londres: Random House.

Medina Girona, D. 20 i 5, "'Poner cera, pulir cera' (filosofía y gamificación)”, Aula. De Innovación Educativa, 246: 26-30.

Prieto Martín, A. et al. 20 I 4, "Experiencias de aplicación de estrategias de gamificación a entornos de aprendizaje universitario", ReVisión, 7, 2, 2014 [http:// www.aenui.net/ojs/index.php?journal=revision\&page=article\&op=view\&pa th[]=149, Consulta: 20 mayo 2016].

Enlaces:

http://www.gamificacion.com/que-es-la-gamificacion [Consulta: mayo 2016] https://en.wikipedia.org/wiki/Gamification [Consulta: mayo 2016] http://jocs.org/ [Consulta: mayo 2016] http://www.oriolripoll.net/ [Consulta: mayo 2016] 